\title{
Combination of intrauterine growth restriction and a high-fat diet impairs cholesterol elimination in rats
}

\author{
Erin K. Zinkhan', Jeanette R. Chin², Jennifer M. Zalla', Baifeng Yu', Ben Numpang ${ }^{1}$, Xing Yu' ${ }^{1}$, Chengshe Jiang', \\ Christopher W. Callaway', Robert A. McKnight', Lisa Joss-Moore' and Robert H. Lane ${ }^{3}$
}

\begin{abstract}
BACKGROUND: Intrauterine growth restriction (IUGR) increases the risk of adult-onset hypercholesterolemia. High-fat diet (HFD) consumption potentiates IUGR-induced increased cholesterol. Cholesterol is converted to bile acids by Cyp7a1 in preparation for excretion. We hypothesized that IUGR rats fed a HFD will have increased cholesterol, decreased Cyp7a1 protein levels, and decreased bile acids compared to control rats fed a HFD.
\end{abstract}

METHODS: At day 21, IUGR and control pups were placed on one of three diets: a regular chow or one of two HFDs containing $1 \%$ or $2 \%$ cholesterol. Cholesterol levels and hepatic Cyp7a1 protein levels were quantified a postnatal week 28.

RESULTS: Both HFDs increased serum cholesterol levels in control rats, and HFD fed IUGR rats had further increased serum cholesterol up to 35-fold. Both HFDs increased hepatic cholesterol levels, and IUGR further increased hepatic cholesterol levels up to fivefold. IUGR decreased hepatic Cyp7a1 protein up to $75 \%$, and hepatic bile acids up to $54 \%$.

CONCLUSION: IUGR increased cholesterol and bile acids and decreased Cyp7a1 protein in rats fed a HFD without changing food intake. These findings suggest that IUGR increases the vulnerability of HFD fed rats to hypercholesterolemia via decreased cholesterol conversion to bile acids.

I ntrauterine growth restriction (IUGR) predisposes to adultonset hypercholesterolemia $(1,2)$. Epidemiologic studies demonstrate that late-gestation exposure to famine makes individuals more susceptible to hypercholesterolemia only when combined with postnatal consumption of a high-fat diet (HFD) (3). These results suggest that the postnatal nutritional environment impacts cholesterol metabolism differently in IUGR individuals compared their normally grown peers. A better understanding of the mechanism through which IUGR combined with a postnatal HFD impacts cholesterol metabolism would provide a foundation to pursue further research into preventing hypercholesterolemia in IUGR individuals.

While the effects of HFD consumption in individuals born IUGR are not well understood, the detrimental effects of HFD consumption in the general population has been extensively documented. HFD consumption often leads to hypercholesterolemia and eventually cardiovascular disease (4). Higher cholesterol levels increase the risk of cardiovascular disease in a continuous, graded fashion $(5,6)$. IUGR may potentiate hypercholesterolemia in individuals that consume a HFD and thus increase morbidity and mortality in this population. The impact of IUGR on cholesterol levels in humans is more evident in the recent decades due to the changing Western diet (7). Average daily fat and cholesterol intake in the United States is $\sim 23-33 \mathrm{~g}$ of saturated fat and up to $400 \mathrm{mg}$ cholesterol, both well above the recommended daily intake of $\sim 16 \mathrm{~g}$ or no more than $7 \%$ of caloric intake for saturated fat and $200 \mathrm{mg}$ cholesterol (7).

The liver is the primary organ for regulating both serum and hepatic cholesterol levels. Cholesterol homeostasis is regulated by a series of hepatic genes. These genes can be organized into four groups, with the key proteins in each group identified in Figure 1. This first group includes cholesterol $7 \alpha$-hydroxylase (Cyp7a1), the rate limiting step of cholesterol conversion to bile acids for elimination from the body. The first group also includes the liver $\mathrm{X}$ receptor $\alpha$ (Lxr $\alpha$ ), the transcription factor both for Cyp7al and for ATP binding cassette transporter a1 (Abcal). The second group contains genes that increase de novo cholesterol synthesis, including peroxisome proliferator receptor $\gamma 1$ (Ppar $\gamma 1)$ and 2 (Ppar 2$)$, sterol responsive element binding protein 2 (Srebp2), and 3-hydroxy-3-methylglutarylCoA reductase (Hmgcr). Group 3 includes the low density lipoprotein (LDL) receptor (Ldlr), which increases cholesterol uptake from the blood, and the Ldlr inhibitor, proprotein convertase subtilisin/kexin type 9 (Pcsk9). The fourth group includes genes involved in high-density lipoprotein (HDL) export, including ATP binding cassette transporters g1 (Abcg1) and Abcal. The final group comprises genes involved with the formation and export of very-low-density lipoprotein cholesterol (VLDL-C), which includes acetyl CoA carboxylase (Acc), fatty acid synthase (Fasn), and microsomal transferase protein (Mtp). Hepatic cholesterol levels provide feedback on key regulators in these pathways to maintain cholesterol

'Division of Neonatology, Department of Pediatrics, University of Utah, Salt Lake City, Utah; ${ }^{2}$ Division of Maternal Fetal Medicine, Department of Obstetrics and Gynecology, University of Utah, Salt Lake City, Utah; ${ }^{3}$ Department of Pediatrics, Medical College of Wisconsin, Milwaukee, Wisconsin. Correspondence: Erin K. Zinkhan (Erin.Zinkhan@hsc.utah.edu) 


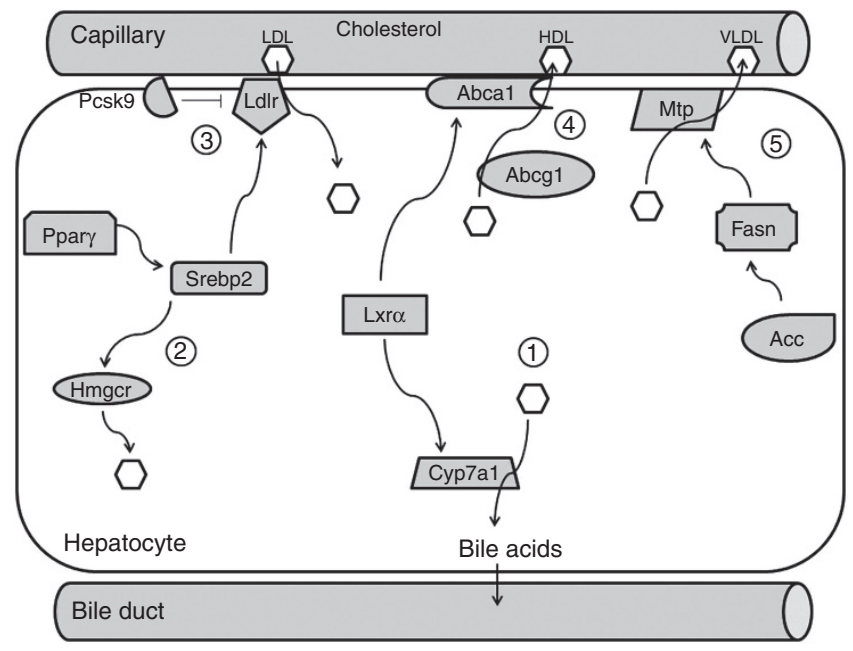

Figure 1. A simplified diagram of major regulatory proteins involved in cholesterol homeostasis is shown here. Group 1 includes Cyp7a1, which catalyzes the rate limiting step in formation of cholic acid, a major bile acid component. Lxr $\alpha$ is a transcription factor for Cyp7a1. Group 2 includes Ppary, a transcription factor for Srebp2. The Srebps are transcription factors for $\mathrm{Hmgcr}$, the rate limiting step in de novo cholesterol synthesis, and Ldlr, the primary means of uptake of LDL-C from the blood. Group 3 consists of Ldlr and Pcsk9, which inhibits Ldlr function. Group 4 includes Abca1 and Abcg1, proteins that function to package cholesterol into HDLC. Group 5 consists of Acc, Fasn, and Mtp, which package triglycerides and cholesterol into VLDL-C for export into the blood.

homeostasis. However, it remains unknown if IUGR followed by postnatal HFD consumption increases hepatic cholesterol and alters expression of cholesterol metabolizing genes more than HFD consumption alone.

The hypothesis of this study is that IUGR rats fed one of two HFDs as depicted in Figure 2 will have increased serum and hepatic cholesterol levels and altered levels of proteins involved in cholesterol synthesis, uptake, and export to VLDL-C and bile acids compared to control rats fed the same HFD.

\section{RESULTS}

\section{Food Intake and Weight Gain}

Rats in both $1 \%$ and $2 \%$ HFD groups consumed fewer grams of food but the same total kilocalories as regular diet fed rats, therefore eating more fat and cholesterol (Figure 3). Rats that consumed the $2 \%$ HFD ate more cholesterol than the rats that consumed the $1 \%$ HFD. No difference was found in fat, cholesterol, or total caloric intake between IUGR and control within any diet.

At weaning on postnatal day 21 IUGR rats weighed less than sex-matched control rats (Supplementary Figure S1 online). IUGR rats caught up to respective diet control rats by week 7. None of the rats exceeded weight gain compared to sexmatched control regular diet rats. All rats had the same mean sex-matched body weight at the time of harvest (week 28).

None of the regular diet fed control or IUGR rat stool samples had increased total or partially digested fat. One of six male IUGR-1\% and two of six female Con-2\% stool samples had increased partially digested fat (not statistically significant compared to Con-Reg).

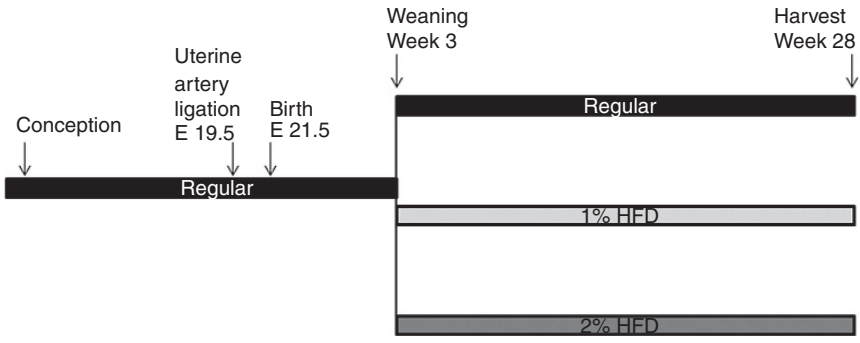

Figure 2. All female rats are kept on the regular diet prior to conception through weaning. Half of female rats undergo bilateral uterine artery ligation at day 19.5 of a 21.5 d gestation to produce intrauterine growth restriction pups. At the time of weaning, postnatal day 21 , the offspring are subsequently weaned to the same maternal regular chow or one of two high-fat diets (HFDs), containing $1 \%$ or $2 \% \mathrm{wt} / \mathrm{wt}$ cholesterol. Offspring are harvested at $28 \mathrm{wk}$ postnatal age.

\section{Serum Cholesterol}

Total cholesterol is comprised of high-density lipoprotein cholesterol (HDL-C) and non-HDL-C. Non-HDL-C is comprised of low-density lipoprotein cholesterol (LDL-C) and very-lowdensity lipoprotein cholesterol (VLDL-C). Lipid panels from HFD female rats were too viscous to accurately calculate LDLC, thus non-HDL-C is shown in Table 1.

No difference was found in serum total or non-HDL cholesterol between control and IUGR rats fed a regular chow. Total and non-HDL cholesterol was increased by both $1 \%$ and $2 \%$ HFDs. IUGR-1\% and IUGR-2\% further increased total and non-HDL cholesterol compared to control rats fed the respective HFD. Serum non-HDL cholesterol levels were significantly higher in female HFD fed rats compared to male HFD fed rats.

Serum HDL cholesterol was unchanged between IUGR and control rats on the regular diet. HDL cholesterol was increased by HFD feeding. Both HFDs also increased HDL cholesterol in both sexes, with no difference between IUGR and control. LDL-C was not able to be calculated due to serum viscosity.

\section{Serum Fatty Acids}

Serum saturated fatty acids were unchanged by IUGR in rats fed a regular diet (Table 2). Both HFDs increased serum saturated fatty acids compared to Con-Reg rats. IUGR combined with either HFD did not alter serum fatty acids compared to control HFD fed rats.

\section{Hepatic Cholesterol}

Hepatic cholesterol was unchanged by IUGR rats fed a regular diet (Table 1). Both HFDs increased hepatic cholesterol levels in males and females compared to Con-Reg rats. IUGR further increased hepatic cholesterol in IUGR-1\% and IUGR$2 \%$ female rats and IUGR-1\% male rats compared to respective Con-HFD rats. Hepatic triglycerides were not consistently increased by IUGR or HFD consumption.

\section{Hepatic Oil Red O Stain}

Both HFDs qualitatively increased hepatic oil red o stain in male and female HFD fed rats (Figure 4). 
a

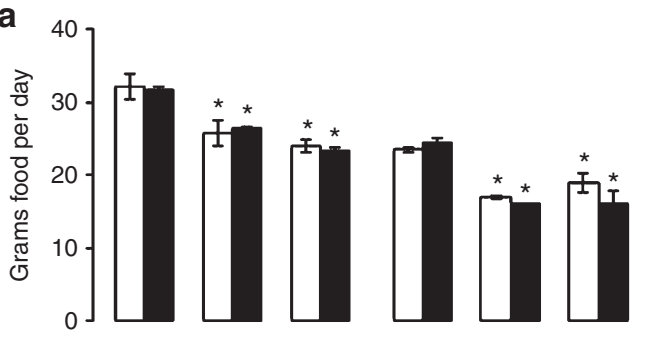

c

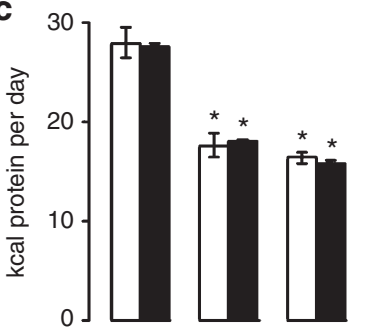

e

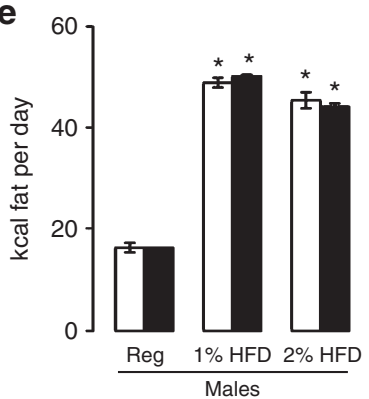

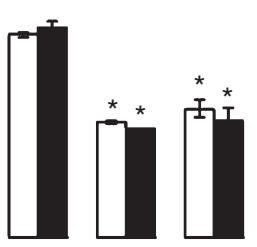

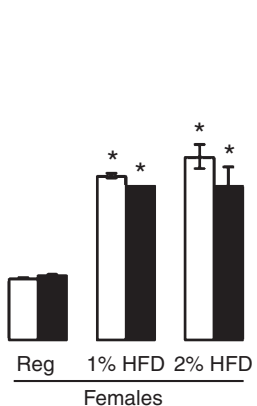

b

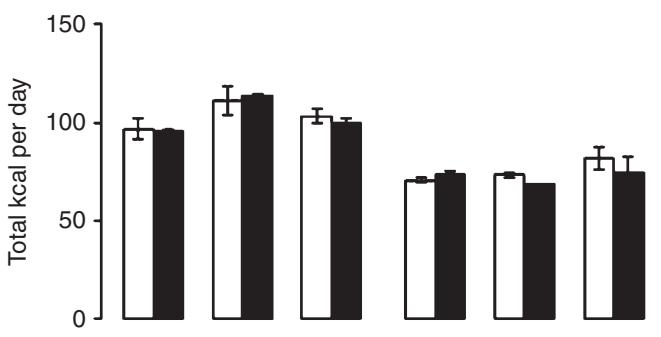

d

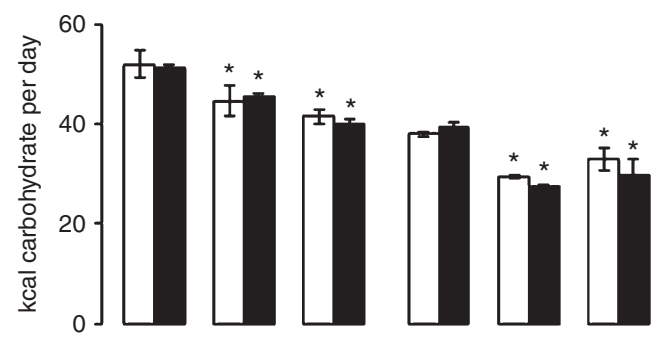

f

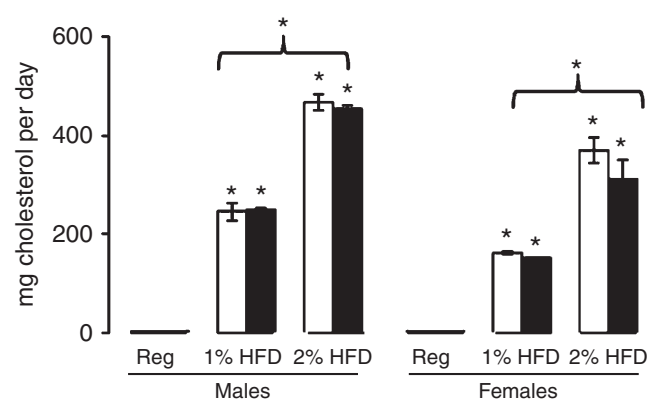

Figure 3. Average food intake over the last 4 wk of the study for regular and HFD fed rats. Diets are not isocaloric. (a) Food intake in grams, (b) total kilocalorie intake, (c) protein intake, (d) carbohydrate intake, (e) fat intake, and (f) cholesterol intake. Rat food was weighed weekly from week 3 through week 28 , and sufficient food supplied to allow ad libatum feeding. Control data are shown in white bars and IUGR data are shown in black bars. Data shown as mean \pm SD. ${ }^{*} P<0.05$ compared to Con-Reg. $N=18$ for male and female regular diet control and IUGR rats, $n=12$ for male and female $1 \%$ HFD control and IUGR rats, and $n=6$ for male and female $2 \%$ HFD control and IUGR rats.

\section{Hepatic Protein}

IUGR combined with either HFD decreased Cyp7a1 protein in IUGR-HFD males and IUGR-2\% females compared to HFD matched controls (Figure 5). IUGR combined with either HFD also decreased Lxr $\alpha$ in males and Lxr $\alpha$ target Abcal in IUGR-1\% males and IUGR-2\% females. IUGR alone minimally affected Cyp7a1, Lxr $\alpha$, Ppar 2 , cleaved Srebp2, cleaved Hmgcr, Ldlr, Pcsk9, Abca1, Abcg1, Acc, Fasn, and Mtp protein levels compared to Con-Reg rats (Figures 6-9). IUGR alone increased Ppar $\gamma 1$ in males and females. HFDs alone as well as IUGR combined with either HFD decreased protein levels of Lxro, Ppar 1 , Ppar 2 , cleaved Srebp2, cleaved Hmgcr, Ldlr, Pcsk9, Abcg1, Acc, Fasn, and Mtp protein compared to ConReg rats.

\section{Hepatic Bile Acids}

IUGR-Reg rats did not have altered hepatic bile acids compared to Con-Reg rats (Table 3). Both HFDs decreased hepatic bile acids in males compared to Con-Reg males. Con-1\% female rats had increased bile acids compared to Con-Reg females. Compared to HFD controls, IUGR-1\% decreased hepatic bile acids in males and females, and IUGR-2\% decreased hepatic bile acids in females.

\section{DISCUSSION}

This study is the first to demonstrate that the combination of IUGR and a postnatal HFD increases cholesterol and decreases hepatic Cyp7al protein and bile acids compared to HFD fed control rats. IUGR combined with a postnatal HFD did not increase proteins involved in de novo cholesterol synthesis, uptake from the blood, or export to the blood. Therefore, increased cholesterol levels may be secondary to decreased Cyp7a1 protein levels and thus decreased conversion of cholesterol to bile acids in rats.

Conversion of cholesterol to bile acids comprises an important step in the regulation of cholesterol homeostasis, with $\sim 50 \%$ of the body's excretion of cholesterol occurring through bile acid formation (8). Cyp7al is the rate limiting and most highly regulated step in bile acid synthesis. Overexpression of Cyp7a1 in cell culture decreases cholesterol levels and increases bile acid synthesis, and has been hypothesized as a treatment modality for hypercholesterolemia $(9,10)$. Conversely, Cyp7a1 deficient mice have higher hepatic and serum cholesterol and decreased total bile acids (11). Importantly, decreased cholesterol conversion to bile acids in the Cyp7a1 knockout mouse is attributable to decreased Cyp7al without compensatory increases in other minor bile acid metabolizing enzymes (12). 
Table 1. Fasting serum lipid panels, markers of liver injury, liver weight as a percentage of body weight, and hepatic lipids

\begin{tabular}{|c|c|c|c|c|c|c|}
\hline & Con-Reg & IUGR-Reg & Con- $1 \%$ & IUGR-1\% & Con- $2 \%$ & IUGR-2\% \\
\hline \multicolumn{7}{|l|}{ Males } \\
\hline Total cholesterol (mg/dl) & $89 \pm 17$ & $85 \pm 14$ & $303 \pm 100^{*}$ & $399 \pm 154^{* \dagger}$ & $222 \pm 101^{*}$ & $339 \pm 111^{*+}$ \\
\hline $\mathrm{HDL}-\mathrm{C}(\mathrm{mg} / \mathrm{dl})$ & $52 \pm 10$ & $53 \pm 7$ & $278 \pm 148^{*}$ & $283 \pm 49 *$ & $231 \pm 120^{*}$ & $160 \pm 66^{*+}$ \\
\hline VLDL-C (mg/dl) & $27 \pm 12$ & $32 \pm 14$ & $21 \pm 7$ & $22 \pm 11$ & $14 \pm 7^{*}$ & $22 \pm 10$ \\
\hline AST (U/I) & $122 \pm 43$ & $117 \pm 35$ & $255 \pm 80^{*}$ & $177 \pm 97^{*+}$ & $239 \pm 69^{*}$ & $238 \pm 75^{*}$ \\
\hline $\operatorname{ALT}(\mathrm{U} / \mathrm{I})$ & $59 \pm 13$ & $59 \pm 13$ & $173 \pm 85^{*}$ & $92 \pm 32^{*+}$ & $127 \pm 42^{*}$ & $147 \pm 63^{*}$ \\
\hline Liver weight:body weight & $3 \% \pm 0.1 \%$ & $3.5 \% \pm 0.1 \%$ & $7.1 \% \pm 0.9 \% *$ & $7.2 \% \pm 0.9 \% *$ & $6.8 \% \pm 0.4 \% *$ & $7.8 \% \pm 0.4 \% *$ \\
\hline Hepatic cholesterol (mg/100 mg liver weight) & $1.5 \pm 0.3$ & $1.6 \pm 0.4$ & $5.4 \pm 0.9^{*}$ & $6.3 \pm 1.7^{*+}$ & $2.6 \pm 0.6^{*}$ & $3.0 \pm 0.4^{*}$ \\
\hline Hepatic triglycerides (nmol/100 mg liver weight) & $35 \pm 17$ & $35 \pm 7$ & $33 \pm 16$ & $31 \pm 5$ & $36 \pm 7$ & $31 \pm 7$ \\
\hline \multicolumn{7}{|l|}{ Females } \\
\hline Non-HDL-C (mg/dl) & $28 \pm 10$ & $32 \pm 13$ & $733 \pm 123^{*}$ & $970 \pm 274^{*+}$ & $528 \pm 277^{*}$ & $960 \pm 621^{*+}$ \\
\hline $\mathrm{HDL}-\mathrm{C}(\mathrm{mg} / \mathrm{dl})$ & $52 \pm 11$ & $50 \pm 6$ & $92 \pm 26^{*}$ & $108 \pm 27^{*}$ & $75 \pm 40^{*}$ & $101 \pm 32^{*}$ \\
\hline VLDL-C (mg/dl) & $20 \pm 17$ & $21 \pm 14$ & $43 \pm 25^{*}$ & $29 \pm 8$ & $24 \pm 10$ & $27 \pm 15$ \\
\hline Triglycerides (mg/dl) & $178 \pm 300$ & $107 \pm 73$ & $299 \pm 238$ & $125 \pm 63$ & $167 \pm 130$ & $135 \pm 76$ \\
\hline AST (U/I) & $143 \pm 122$ & $86 \pm 17$ & - & - & - & - \\
\hline $\operatorname{ALT}(\mathrm{U} / \mathrm{I})$ & $88 \pm 72$ & $51 \pm 5$ & - & - & - & $-{ }^{*}$ \\
\hline Liver weight: body weight & $2.9 \% \pm 0.1 \%$ & $2.9 \% \pm 0.4 \%$ & $9.8 \% \pm 0.4 \% *$ & $9.6 \% \pm 0.5 \% *$ & $9.7 \% \pm 0.4 \% *$ & $9.5 \% \pm 0.4 \% *$ \\
\hline Hepatic cholesterol (mg/100 mg liver weight) & $1.4 \pm 0.1$ & $1.8 \pm 0.2$ & $7.0 \pm 2.3^{*}$ & $11 \pm 2.8^{*+}$ & $3.6 \pm 0.6^{*}$ & $6.8 \pm 1.8^{*+}$ \\
\hline Hepatic triglycerides ( $\mathrm{nmol} / 100 \mathrm{mg}$ liver weight) & $16 \pm 6$ & $22 \pm 6$ & $20 \pm 7$ & $17 \pm 12$ & $23 \pm 5$ & $27 \pm 3^{*}$ \\
\hline
\end{tabular}

IUGR induced by protein restriction decreases Cyp7a1, histone acetylation and RNA polymerase II in the Cyp7a1 promoter, and increased serum and hepatic cholesterol in adult male rats (13). This finding suggests that Cyp7a1 is a gene susceptible to the in utero nutritional environment resulting in long-term decreases in expression and cholesterol accumulation. Interestingly in our model IUGR in combination with a HFD did not alter de novo cholesterol synthesis, LDL-C uptake from the blood, or export of HDL-C or VLDL-C to the blood, and only decreased cholesterol elimination in bile. While decreased conversion of cholesterol to bile acids is unlikely to be the only mechanism through which IUGR increased cholesterol, the decreased Cyp7a1 protein levels observed in our study likely represents a major pathway through which IUGR adversely modulates cholesterol homeostasis.

HFD feeding, which is common in developed nations today, leads to hypercholesterolemia and liver injury. In our study, HFD feeding alone increased serum and hepatic cholesterol. Similarly, HFD feeding in mice increased hepatic cholesterol compared to low-fat diet controls (14). Furthermore, HFD feeding alone increased markers of hepatic injury, as seen with oil red o staining and enzyme markers of hepatic injury in our study, with no difference between IUGR-HFD and Con-HFD. Similarly, maternal hypoxia increased fatty liver in HFD fed mice (15). HFD feeding alone decreased proteins involved in de novo cholesterol synthesis, LDL-C uptake from blood, and export of HDL-C and VLD-C export to the blood. While it is well accepted that HFD feeding alone is detrimental to health, the impact of IUGR combined with HFD feeding has been little studied.

Our study showed increased serum non-HDL cholesterol in IUGR and control females relative to males. IUGR induces sex-specific differences in cholesterol metabolism in both humans and other rodent models $(2,16)$. Human data demonstrate a greater LDL-C to HDL-C ratio in men compared to women who were IUGR at birth (3). Sex specific responses in cholesterol metabolizing genes are seen in Cyp7a1 knockout mice fed cholesterol. Female Cyp7a1 knockout mice have a threefold increase in hepatic cholesterol and increased cholesterol absorption while males have minimal increases in cholesterol absorption and hepatic cholesterol (12). Higher cholesterol was found in female compared to male LDL-receptor knockout mice fed a low-fat chow (17). Furthermore, orchidectomy increased cholesterol in males but ovariectomy did not alter 


\section{Articles | Zinkhan et al.}

Table 2. Fasting serum free fatty acids

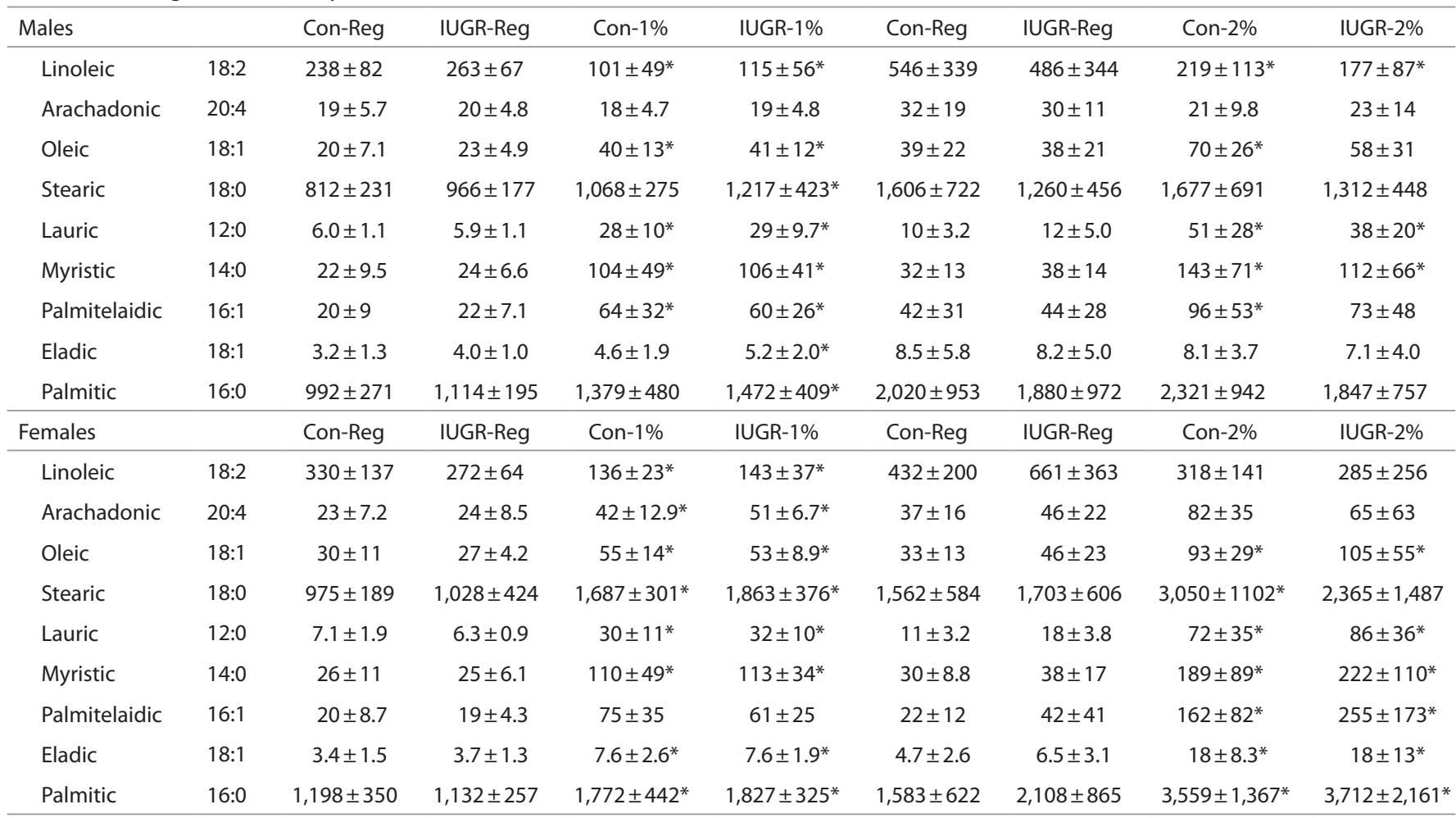

Frozen serum was analyzed with mass spectrometry for fatty acid quantification. Lipid carbon number and double bond designation is shown next to each lipid name. Data shown as area under the curve \pm SD. $N=6$ for each group. Con-Reg and IUGR-Reg data shown twice, as mass spectrometry data were run at different times along with serum from rats consuming HFD $1 \%$ and HFD 2\%, and drift in the mass spectrometry equipment necessitates controls with each run.

${ }^{*} P<0.05$ compared to sex-matched Con-Reg.
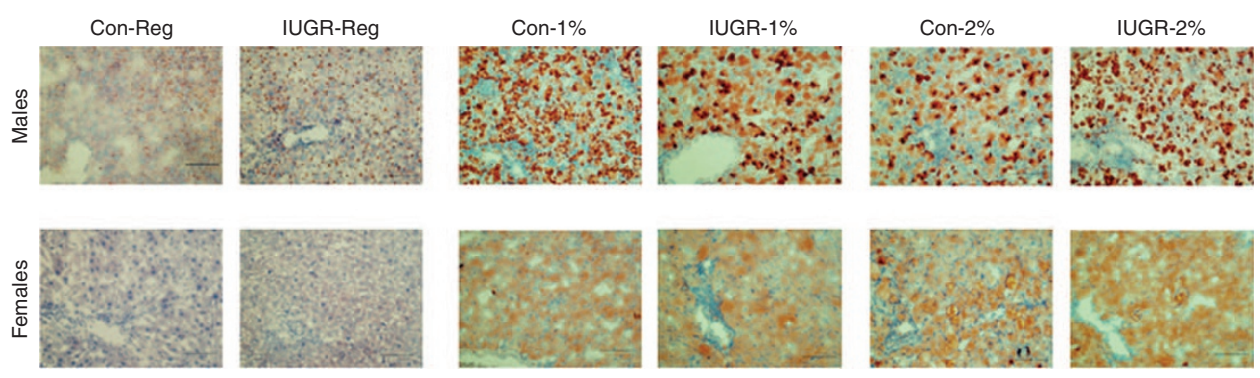

Figure 4. Hepatic Oil Red O stain of fresh frozen livers demonstrated increased hepatic fat stain in HFD fed rats. Scale bar represents $50 \mu \mathrm{m}$ and images are taken at $80 \times$ magnification. Livers frozen at necropsy in Optimum Cutting Temperature, sliced with a thickness of $6 \mu \mathrm{m}$, and stained with Oil Red $\mathrm{O}$ and hematoxylin. $N=4$ for each group.

cholesterol in females (17). Androgen levels may be protective for hypercholesterolemia, and dietary fat and cholesterol may interplay with sex steroids to alter cholesterol metabolism (17).

The specific etiology of the IUGR insult differentially impacts the formation of hypercholesterolemia. While nutrient restriction is the most common cause of IUGR in the developing world, uteroplacental insufficiency is the most common intrauterine insult leading to IUGR in the developed world, where HFD consumption is common (18). Uteroplacental insufficiency induced IUGR produces and intrauterine mileu of hypoglycemia, hypoxia, and acidosis in the IUGR fetus (19). The type of IUGR insult used in this study replicates this intrauterine mileu in the fetal pups $(20,21)$.
The impact of IUGR on cholesterol metabolism has been studied using other models of IUGR. Other rodent studies using an IUGR insult induce IUGR either through hypoxia or maternal micro- or macronutrient restriction $(16,22,23)$. In contrast to our study, hypoxia and nutrient restriction IUGR insults produce elevated serum and hepatic cholesterol levels when fed a regular, healthy diet. When hypoxia-induced IUGR rats were subsequently fed a HFD without added cholesterol, IUGR did not increase serum or hepatic cholesterol compared to HFD fed control rats (24). In our study, uteroplacental insufficiency induced IUGR increased serum and hepatic cholesterol accumulation only in combination with a second hit, the consumption of a HFD. 

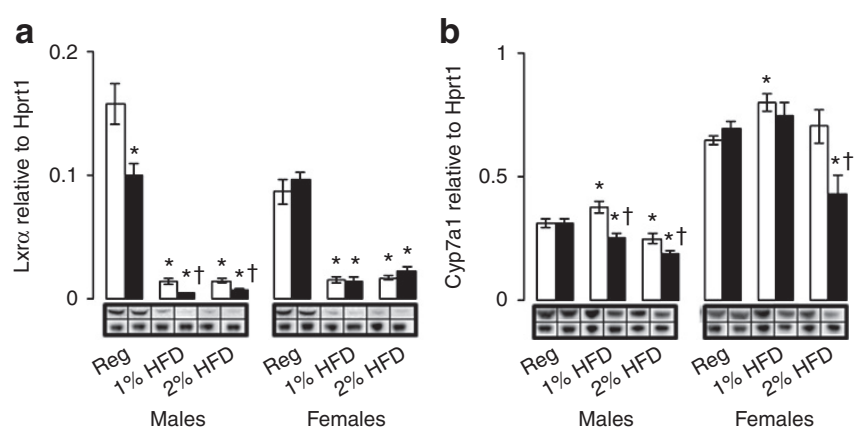

Figure 5. Hepatic protein levels for group 1. (a) Lxro and (b) Cyp7a1 protein. Data analyzed using western blot normalized to Hprt1. Control data are shown in white bars and IUGR data are shown in black bars. Data shown as mean \pm SD. Western blot images correspond to the above bar graph, with protein of interest on the top and Hprt1 below. $N=6,{ }^{*} P<0.05$ compared to Con-Reg, and ${ }^{+} P<0.05$ compared to respective Con-HFD.
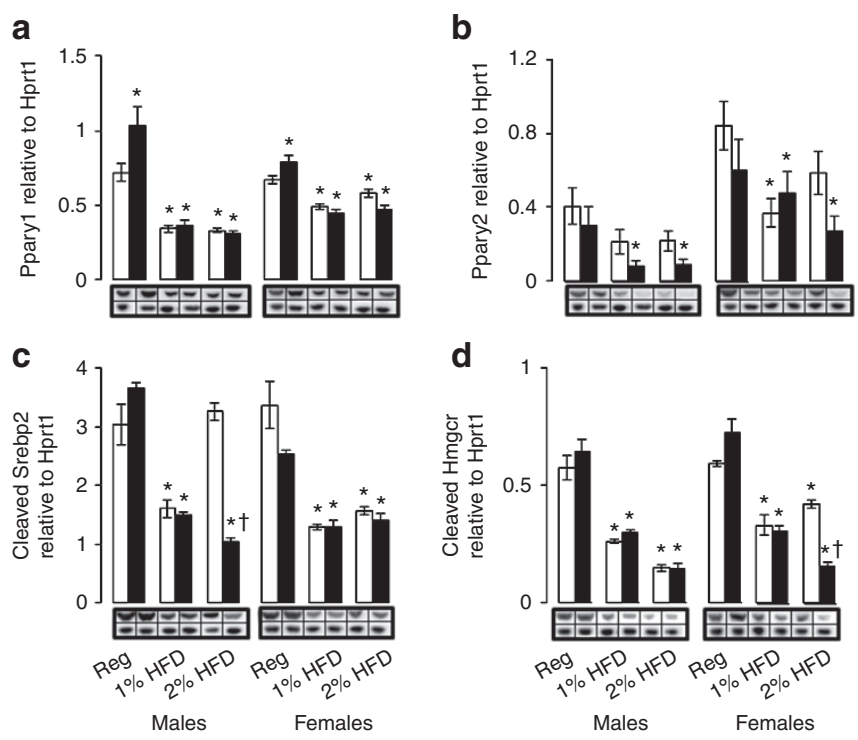

Figure 6. Hepatic protein levels for group 2. (a) Ppary1, (b) Ppary2, (c) cleaved Srebp2, and (d) cleaved Hmgcr protein. Data analyzed using western blot normalized to Hprt1. Control data are shown in white bars and IUGR data are shown in black bars. Data shown as mean \pm SD. Western blot images correspond to the above bar graph, with protein of interest on the top and Hprt1 below. $N=6,{ }^{*} P<0.05$ compared to Con-Reg, and ${ }^{\dagger} P<$ 0.05 compared to respective Con-HFD.

Alteration of free fatty acid and triglyceride levels also depend on the etiology of IUGR insult. Both HFDs increased serum saturated fatty acids and decreased the omega- 6 fatty acid linoleic acid in this study, consistent with increased dietary intake of saturated fat from milk fat and decreased soybean oil (Table 2). However, IUGR-1\% and IUGR-2\% increased serum cholesterol levels without significant changes in triglyceride and serum free fatty acids (Tables 1 and 2). Consistent with our study, triglyceride levels were unchanged in IUGR infants exposed to the Dutch famine during the last third of gestation (25). Other epidemiologic studies demonstrate that IUGR alters serum triglyceride levels (1). However, many epidemiologic studies utilize small for gestational age (SGA) as a marker of IUGR. Many SGA infants are not growth restricted a

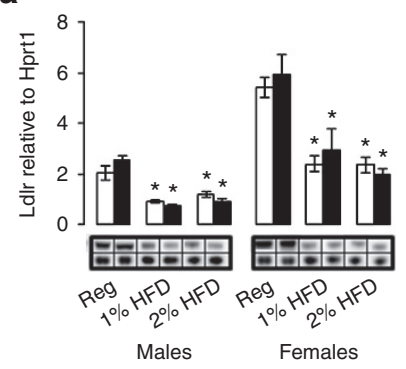

b

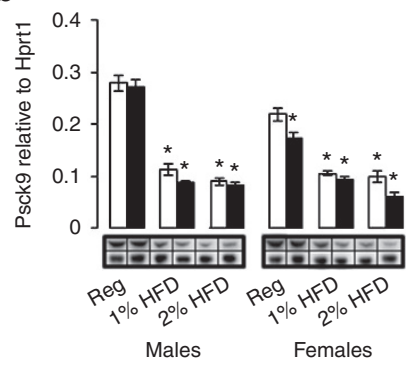

Figure 7. Hepatic protein levels for group 3. (a) Ldlr and (b) Pcsk9 protein. Data analyzed using western blot normalized to Hprt1. Control data are shown in white bars, and IUGR data are shown in black bars. Data shown as mean \pm SD. Western blot images correspond to the above bar graph, with protein of interest on the top and Hprt1 below. $N=6,{ }^{*} P<0.05$ compared to Con-Reg.
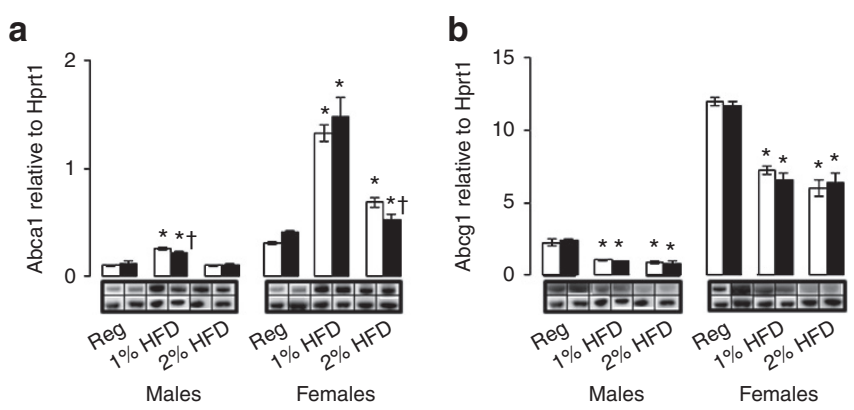

Figure 8. Hepatic protein levels for group 4. (a) Abca1 and (b) Abcg1 protein. Data analyzed using western blot normalized to Hprt1. Control data are shown in white bars and IUGR data are shown in black bars. Data shown as mean \pm SD. Western blot images corresponds to the above bar graph, with protein of interest on the top and Hprt1 below. $N=6,{ }^{*} P<0.05$ compared to Con-Reg, and ${ }^{t} P<0.05$ compared to respective Con-HFD.

and thus not likely predisposed to abnormal lipid metabolism. When there is an etiology for probable growth restriction, such as during a famine, the timing, duration, and specific maternal micro- and macro-nutrient availability at the time of the famine may explain the discrepancies between these studies.

Consistent with human studies, animal studies indicate that hypoxia and nutrition restriction induced IUGR either increase or do not change serum triglyceride levels $(16,22)$. IUGR caused by uteroplacental insufficiency increased serum triglycerides in $120 \mathrm{~d}$ old but not $21 \mathrm{~d}$ old IUGR rats, time points which were not examined in our study (26). Increased cholesterol without increased triglycerides or free fatty acids as seen in this study suggests that IUGR specifically alters the regulation of cholesterol metabolizing genes rather than globally altering fatty acid metabolism (27).

A limitation of our study is the decreased protein intake of the HFD animals compared to the regular diet rats. The effect of decreased protein consumption on our results cannot be excluded, however, the protein content was chosen to be similar to the typical American diet, is sufficient for normal mammalian growth, and is significantly higher than protein content used in low protein studies $(7,28,29)$. Furthermore, caution should always be used in relating animal data to humans.

In conclusion, in rats fed a HFD, IUGR increased serum and hepatic cholesterol without changing fat or cholesterol 

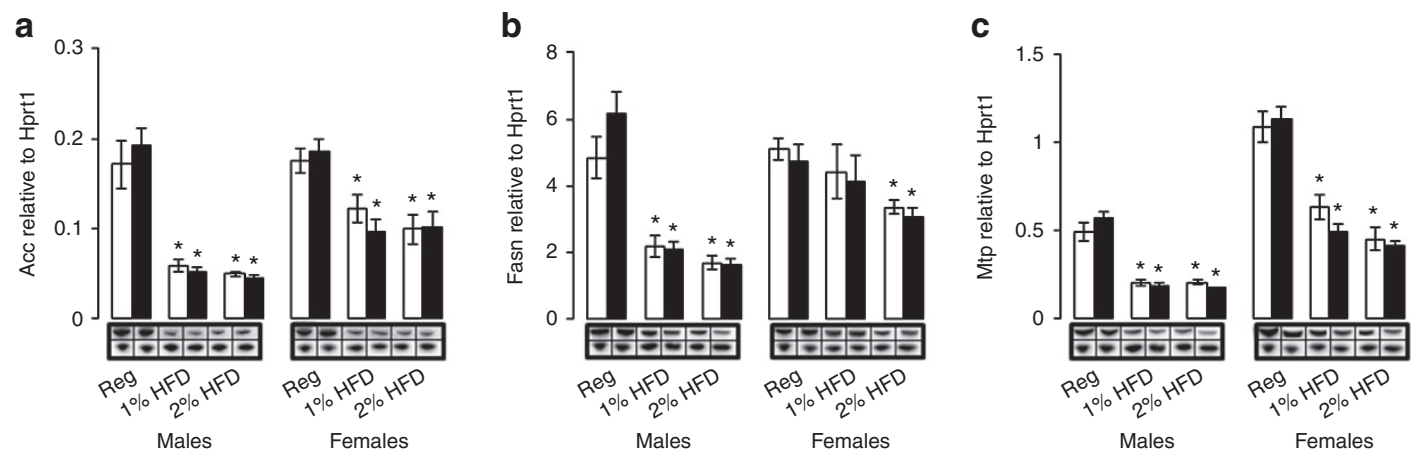

Figure 9. Hepatic protein levels for group 5. (a) Acc, (b) Fasn, and (c) Mtp protein. Data analyzed using western blot normalized to Hprt1. Control data are shown in white bars, and IUGR data are shown in black bars. Data shown as mean \pm SD. Western blot images correspond to the above bar graph, with protein of interest on the top and Hprt1 below. $N=6,{ }^{*} P<0.05$ compared to Con-Reg.

Table 3. Fasting hepatic bile acids

\begin{tabular}{lcccccc}
\hline & \multicolumn{3}{c}{ IUGR- } & & & \\
& Con-Reg & Reg & Con-1\% & IUGR-1\% & Con-2\% & IUGR-2\% \\
\hline Males & $14 \pm 3.2$ & $12 \pm 1.7$ & $5.4 \pm 1.8^{*}$ & $1.1 \pm 0.6^{*+}$ & $2.4 \pm 0.7^{*}$ & $1.3 \pm 0.6^{*}$ \\
Females & $3.2 \pm 0.6$ & $2.2 \pm 0.5$ & $6.9 \pm 1.5^{*}$ & $3.1 \pm 0.4^{\dagger}$ & $3.9 \pm 0.4$ & $2.0 \pm 0.8^{\dagger}$
\end{tabular}

Hepatic bile acids were isolated and quantified with a colorimetric kit. $N=6$.

${ }^{*} P<0.05$ compared to Con-Reg. ${ }^{+} P<0.05$ compared to respective Con-HFD.

consumption. IUGR decreased hepatic Cyp7a1 protein levels and bile acids in HFD fed rats. These findings suggest that IUGR adversely decreases cholesterol conversion to bile acids, a major pathway through which cholesterol is excreted from the body, leading to decreased cholesterol elimination. A better understanding of the mechanism through which IUGR combined with a postnatal HFD decreases cholesterol elimination may lead to tailored treatments for IUGR individuals.

\section{METHODS}

Animals

The University of Utah Animal Care Committee approved all animal procedures. Timed pregnant female Sprague Dawley rats were obtained from Charles River Laboratories (Wilmington, MA). Bilateral uterine artery ligation was performed on day 19.5 of a 21.5 d gestation to produce $20-25 \%$ growth restricted rats as described previously (30). Rats were allowed to deliver naturally and litters were culled to six at birth for rearing consistency. A total of 12 dams underwent uterine artery ligation and 12 dams received anesthesia alone as a control.

The quantity of cholesterol consumption alters serum cholesterol levels (31), therefore at weaning on postnatal week 3 IUGR and control offspring were started on one of three diets, detailed in Figure 2. This experimental design yielded a total of 144 offspring rats, with an $n$ of 6-18 rats per sex, diet, and intrauterine environment group. Siblings from the same mother were not used in the same experiments unless they were of a different sex or on a different diet. This resulted in 12 groups, 6 groups for each sex: control rats fed a regular diet (Con-Reg), control rats fed a 1\% HFD (Con-1\%), control rats fed a $2 \%$ HFD (Con-2\%), IUGR rats fed a regular diet (IUGR-Reg), IUGR rats fed a $1 \%$ HFD (IUGR-1\%), and IUGR rats fed a $2 \%$ HFD (IUGR-2\%).

The diets used in this study are a regular rat chow (Reg, HarlanTeklad, TD.8640, Madison, WI), 1\% HFD (Harlan Teklad, TD.110526), or 2\% HFD (Harlan Teklad, TD.110197). The regular diet contained $17 \% \mathrm{kcal}$ from fat, $54 \% \mathrm{kcal}$ carbohydrate, and $29 \% \mathrm{kcal}$ from protein. The fat source in the regular rat chow was from $60 \mathrm{~g} / \mathrm{kg}$ soybean oil and contains $0.03 \% \mathrm{wt} / \mathrm{wt}$ cholesterol. Both $1 \%$ and $2 \%$ HFDs contain $44 \% \mathrm{kcal}$ from fat, $40 \% \mathrm{kcal}$ from carbohydrate, and $16 \% \mathrm{kcal}$ from protein. The fat source in both $1 \%$ and $2 \%$ HFDs was milk fat and $10 \mathrm{~g} / \mathrm{kg}$ soybean oil, consisting of $65 \%$ saturated fat. The $1 \%$ HFD contains $1 \% \mathrm{wt} / \mathrm{wt}$ cholesterol and the $2 \%$ HFD contains $2 \% \mathrm{wt} / \mathrm{wt}$ cholesterol. Both HFDs also contain $0.5 \%$ cholic acid to enable intestinal absorption of fat and cholesterol. Protein content in both HFDs is lower than the Reg diet, though was chosen to mimic protein consumption in the United States today (7) and is sufficient for normal mammalian growth (29). Furthermore, this protein content is significantly higher than protein content used in low protein dietary studies (28).

All rats and food were weighed weekly and rats were not pair fed because preliminary studies demonstrated that rats on all three diets consumed the same total kilocalories as regular diet fed controls. Diets were continued until adult IUGR and control rats were harvested at postnatal week 28 . Rats were fasted for $6-8 \mathrm{~h}$ prior to harvest, and all liver and serum results were obtained on fasted liver and serum. At harvest, rats were anesthetized with isoflurane prior to decapitation. Mature females were harvested only in estrus to minimize confounders of the estrus cycle on cholesterol metabolism (32). Fresh stool was collected from $n=6$ non-fasting rats from each group and sent to ARUP laboratories for qualitative fecal fat (ARUP lab number 0020385), separating fat into total fat and partially digested fat.

\section{Serum Cholesterol Analysis}

Mixed arterial and venous serum was collected at necropsy in serum separator tubes (BD Vacutainer, BD, Franklin Lakes, NJ) and allowed to clot at room temperature prior to separation. Serum aliquots were frozen at $-80{ }^{\circ} \mathrm{C}$. Serum lipid panels were analyzed at ARUP laboratories within $1 \mathrm{wk}$ of collection (ARUP lab number 0020421). To look for markers of liver inflammation, serum was also sent to ARUP for aspartate transaminase (AST) or alanine transaminase (ALT) analysis (ARUP lab number 0020408).

To determine specificity of IUGR on serum cholesterol, serum fatty acid concentrations were also measured. Gas chromatography-mass spectrometry was performed to determine serum fatty acid levels. Because GC-MS data are represented in arbitrary units and comparisons are performed on control samples run at the same time, data are represented as a percent of sex- and age-matched control rat serum level. Serum fatty acid analysis was performed using GC-MS (Waters GCT Premier mass spectrometer, Milford, MA) with an Agilent 6890 gas chromatograph (Hewlett Packard, Ramsey, MN) and a Gerstel MPS2 autosampler (Linthicum, MD) at the metabolomics core facility at the University of Utah.

\section{Hepatic Cholesterol Quantification}

Liver lipid was isolated based on a method developed by Folch et al. (33). Lipid was quantified using a colorimetric kit for cholesterol (BioVision HDL-C and LDL-C/VLDL-C Cholesterol Quantification Kit, Biovision Research Products, Mountain View, CA) and for triglyceride (BioVision Triglyceride Quantification Kit, BioVision Research Products), according to manufacturers' protocol. Data was calculated as $\mathrm{mg}$ cholesterol or nmol triglyceride/100 g liver weight and compared to reference data. 


\section{IUGR-HFD impairs cholesterol elimination}

\section{Hepatic Oil Red O Stain}

At necropsy livers were weighed and a small piece of the left anterior lobe of the liver was cut and placed on ice prior to fresh embedding in Optimal Cutting Temperature (VWR, Radnor, PA). Embedded livers were sliced to $6 \mu \mathrm{m}$, fixed with $10 \%$ formalin to a glass slide, washed with $100 \%$ propylene glycol (ACROS Organics, Thermo Fisher Scientific, Fair Lawn, NJ), stained with Oil Red O (Amresco, Solon, $\mathrm{OH}$ ), washed with $85 \%$ propylene glycol, stained with Gill \#2 modified hematoxylin, and mounted with Aquamount (Thermo Scientific, Kalamazoo, MI). All images were stained and visualized at the same time as their respective controls using a light microscope at $80 \times$ magnification. The bars in Figure 4 represent $50 \mu \mathrm{m}$.

\section{Hepatic Protein Quantification}

Hepatic protein was isolated using whole cell lysates in buffer consisting of $150 \mathrm{mmol} / \mathrm{l} \mathrm{NaCl}, 50 \mathrm{mmol} / \mathrm{l}$ Tris $\mathrm{pH} 7.4,1 \mathrm{mmol} / \mathrm{l} \mathrm{EDTA}, 0.25 \%$ $\mathrm{Na}$-deoxycholate, $1 \%$ Igepal CA-630. Total protein concentrations were determined bicinchoninic acid assay with BSA as a standard curve. Fifty micrograms protein was run on a $10 \%$ SDS PAGE gel (Bio-Rad, Hercules, CA). The protein was then transferred to a PVDF membrane and blocked with 5\% milk. Hprt1 (Proteintech, Chicago, IL) was used as a loading control, as Hprtl did not differ between intrauterine environment and dietary administration. Antibodies used were Cyp7a1 (Santa Cruz Biotechnology, Dallas, TX), Lxro (LifeSpan BioSciences), Ppary (Santa Cruz Biotechnology), Srebp2 (Abcam), Hmgcr (Novus Biologicals, Littleton, CO), Ldlr (BioVision, Milpitas, CA), Pcsk9 (LifeSpan BioSciences, Seattle, WA), Abcal (Novus Biologicals), Abcg1 (Santa Cruz Biotechnology), Acc (Cell Signaling Technology, Danvers, MA), Fasn (BD Transduction Laboratories, Santa Cruz, CA), and Mtp (BD Transduction Laboratories). Western Lightning enhanced chemiluminescence (ECL) (PerkinElmer Life Sciences) was used to detect protein using goat anti-rabbit or anti-mouse (for Fasn and Mtp) horseradish peroixidase conjugated secondary antibody from Cell Signaling Technology. A Kodak Image Station 2000ER (Eastman Kodak/SIS, Rochester, NY) was used to image and quantify protein.

Due to the large number of samples for comparison, control and IUGR regular diet male (or female) samples were run on every gel, and either $1 \%$ HFD or $2 \%$ HFD control and IUGR samples run on the other half of the gel. Each target protein was compared to Hprt1 protein for that sample. Regular diet control data from the 2\% HFD gel were compared to regular diet control data from the 1\% HFD gel, and the 2\% HFD data normalized for differences between regular diet control data. Male and female samples were not run on the same gel and thus cannot be directly compared. Given the large number of samples, the western blot images were cropped from the gels but not otherwise altered.

\section{Hepatic Bile Acid Quantification}

Rats do not have gall bladders; therefore hepatic bile acids were measured as a marker of Cyp7al activity. Hepatic bile acids were extracted from $100 \mathrm{mg}$ frozen liver tissue crushed over liquid nitrogen as per a user supplied protocol from Crystal Chem (Downers Grove, IL). Crushed tissue was resuspended in $1 \mathrm{ml} 75 \%$ ethanol for $2 \mathrm{~h}$ at $50{ }^{\circ} \mathrm{C}$. Twenty microliters supernatant was used in a Rat Total Bile Acids kit (Crystal Chem) per manufacturer's instructions with additional bile acid controls (Crystal Chem). Male and female bile acid samples were run on separate plates and thus not directly comparable.

\section{Statistics}

Data tables and figures were expressed as mean \pm SD. Analysis of variance (Fisher's protected least-significant difference) and Student's unpaired $t$-test were used for data analysis. Binomial data was analyzed using Fisher's exact test. A $P$ value $<0.05$ was considered to be statistically significant.

\section{SUPPLEMENTARY MATERIAL}

Supplementary material is linked to the online version of the paper at http://www.nature.com/pr

\section{ACKNOWLEDGMENTS}

We thank Amnon Schlegel, M.D., Ph.D., at the University of Utah for supplying antibodies and scientific direction. This research was conducted at the University of Utah, Salt Lake City, UT.

\section{STATEMENT OF FINANCIAL SUPPORT}

No funding sources were used to prepare data presented in this article. Disclosure: There is no conflict of interest to disclose.

\section{REFERENCES}

1. Barker DJ, Martyn CN, Osmond C, Hales CN, Fall CH. Growth in utero and serum cholesterol concentrations in adult life. BMJ 1993;307: $1524-7$.

2. Forsdahl A. Living conditions in childhood and subsequent development of risk factors for arteriosclerotic heart disease. The cardiovascular survey in Finnmark 1974-75. J Epidemiol Community Health 1978;32:34-7.

3. Robinson SM, Batelaan SF, Syddall HE, et al.; Hertfordshire Cohort Study. Combined effects of dietary fat and birth weight on serum cholesterol concentrations: the Hertfordshire Cohort Study. Am J Clin Nutr 2006;84:23744.

4. Spinler SA, de Denus S, Earl G, Cheng JW. Plasma cholesterol concentrations, dietary fat intake, and cholesterol intake in pharmacy students. J Am Pharm Assoc (2003) 2003;43:590-5.

5. Neaton JD, Blackburn H, Jacobs D, et al. Serum cholesterol level and mortality findings for men screened in the Multiple Risk Factor Intervention Trial. Multiple Risk Factor Intervention Trial Research Group. Arch Intern Med 1992;152:1490-500.

6. Stamler J, Daviglus ML, Garside DB, Dyer AR, Greenland P, Neaton JD. Relationship of baseline serum cholesterol levels in 3 large cohorts of younger men to long-term coronary, cardiovascular, and all-cause mortality and to longevity. JAMA 2000;284:311-8.

7. Wright JD, Wang CY, Kennedy-Stephenson J, Ervin RB. Dietary intake of ten key nutrients for public health, United States: 1999-2000. Adv Data 2003;334:1-4.

8. Vlahcevic ZR, Pandak WM, Stravitz RT. Regulation of bile acid biosynthesis. Gastroenterol Clin North Am 1999;28:1-25, v.

9. Spady DK, Cuthbert JA, Willard MN, Meidell RS. Adenovirus-mediated transfer of a gene encoding cholesterol 7 alpha-hydroxylase into hamsters increases hepatic enzyme activity and reduces plasma total and low density lipoprotein cholesterol. J Clin Invest 1995;96:700-9.

10. Pandak WM, Schwarz C, Hylemon PB, et al. Effects of CYP7A1 overexpression on cholesterol and bile acid homeostasis. Am J Physiol Gastrointest Liver Physiol 2001;281:G878-89.

11. Erickson SK, Lear SR, Deane S, et al. Hypercholesterolemia and changes in lipid and bile acid metabolism in male and female cyp7A1-deficient mice. J Lipid Res 2003;44:1001-9.

12. Schwarz M, Russell DW, Dietschy JM, Turley SD. Alternate pathways of bile acid synthesis in the cholesterol 7alpha-hydroxylase knockout mouse are not upregulated by either cholesterol or cholestyramine feeding. J Lipid Res 2001;42:1594-603.

13. Sohi G, Marchand K, Revesz A, Arany E, Hardy DB. Maternal protein restriction elevates cholesterol in adult rat offspring due to repressive changes in histone modifications at the cholesterol 7alpha-hydroxylase promoter. Mol Endocrinol 2011;25:785-98.

14. Heinonen I, Rinne P, Ruohonen ST, Ruohonen S, Ahotupa M, Savontaus E. The effects of equal caloric high fat and western diet on metabolic syndrome, oxidative stress and vascular endothelial function in mice. Acta Physiol (Oxf) 2014;211:515-27.

15. Su YM, Lv GR, Xie JX, Wang ZH, Lin HT. Maternal hypoxia increases the susceptibility of adult rat male offspring to high-fat diet-induced nonalcoholic fatty liver disease. Endocrinology 2013;154:4377-87.

16. Sohi G, Revesz A, Hardy DB. Permanent implications of intrauterine growth restriction on cholesterol homeostasis. Semin Reprod Med 2011;29:246-56

17. Hatch NW, Srodulski SJ, Chan HW, Zhang X, Tannock LR, King VL. Endogenous androgen deficiency enhances diet-induced hypercholesterolemia and atherosclerosis in low-density lipoprotein receptor-deficient mice. Gend Med 2012;9:319-28.

18. Kinzler WL, Vintzileos AM. Fetal growth restriction: a modern approach. Curr Opin Obstet Gynecol 2008;20:125-31. 


\section{Articles | Zinkhan et al.}

19. Nieto-Díaz A, Villar J, Matorras-Weinig R, Valenzuela-Ruìz P. Intrauterine growth retardation at term: association between anthropometric and endocrine parameters. Acta Obstet Gynecol Scand 1996;75:127-31.

20. Ogata ES, Bussey ME, Finley S. Altered gas exchange, limited glucose and branched chain amino acids, and hypoinsulinism retard fetal growth in the rat. Metab Clin Exp 1986;35:970-7.

21. Ogata ES, Bussey ME, LaBarbera A, Finley S. Altered growth, hypoglycemia, hypoalaninemia, and ketonemia in the young rat: postnatal consequences of intrauterine growth retardation. Pediatr Res 1985;19:32-7.

22. Choi GY, Tosh DN, Garg A, Mansano R, Ross MG, Desai M. Genderspecific programmed hepatic lipid dysregulation in intrauterine growthrestricted offspring. Am J Obstet Gynecol 2007;196:477.e1-7.

23. Kris-Etherton PM, Layman DK, York PV, Frantz ID Jr. The influence of early nutrition on the serum cholesterol of the adult rat. J Nutr 1979;109:1244-57.

24. Rueda-Clausen CF, Dolinsky VW, Morton JS, Proctor SD, Dyck JR, Davidge ST. Hypoxia-induced intrauterine growth restriction increases the susceptibility of rats to high-fat diet-induced metabolic syndrome. Diabetes 2011;60:507-16.

25. Roseboom TJ, van der Meulen JH, Osmond C, Barker DJ, Ravelli AC, Bleker OP. Plasma lipid profiles in adults after prenatal exposure to the Dutch famine. Am J Clin Nutr 2000;72:1101-6.

26. Lane RH, Kelley DE, Gruetzmacher EM, Devaskar SU. Uteroplacental insufficiency alters hepatic fatty acid-metabolizing enzymes in juvenile and adult rats. Am J Physiol Regul Integr Comp Physiol 2001;280:R18390.

27. Kind KL, Clifton PM, Katsman AI, Tsiounis M, Robinson JS, Owens JA. Restricted fetal growth and the response to dietary cholesterol in the guinea pig. Am J Physiol 1999;277(6 Pt 2):R1675-82.

28. Boujendar S, Reusens B, Merezak S, et al. Taurine supplementation to a low protein diet during foetal and early postnatal life restores a normal proliferation and apoptosis of rat pancreatic islets. Diabetologia 2002;45:856-66.

29. 1973 Energy and protein requirements. Report of a joint FAO/WHO ad hoc expert committee. Rome, 22 March-2 April 1971. FAO Nutr Meet Rep 1973;522:1-118.

30. Fu Q, McKnight RA, Yu X, Callaway CW, Lane RH. Growth retardation alters the epigenetic characteristics of hepatic dual specificity phosphatase 5. FASEB J 2006;20:2127-9.

31. Singhal A, Cole TJ, Fewtrell M, Lucas A. Breastmilk feeding and lipoprotein profile in adolescents born preterm: follow-up of a prospective randomised study. Lancet 2004;363:1571-8.

32. Hubscher CH, Brooks DL, Johnson JR. A quantitative method for assessing stages of the rat estrous cycle. Biotech Histochem 2005;80:79-87.

33. Folch J, Lees M, Sloane Stanley GH. A simple method for the isolation and purification of total lipides from animal tissues. J Biol Chem 1957;226:497-509. 